\title{
Association of Gestational Diabetes Mellitus with Adverse Pregnancy Outcomes and Its Interaction with Maternal Age in Chinese Urban Women
}

\author{
Xueyin Wang $\mathbb{D}^{\mathbb{2}}$, Xiaosong Zhang, Min Zhou, Juan Juan, and Xu Wang \\ Department of Obstetrics and Gynecology, Peking University First Hospital, Beijing 10034, China \\ Correspondence should be addressed to Xueyin Wang; xueyin0925@163.com
}

Received 6 January 2021; Accepted 7 May 2021; Published 19 May 2021

Academic Editor: Ulrike Rothe

Copyright (C) 2021 Xueyin Wang et al. This is an open access article distributed under the Creative Commons Attribution License, which permits unrestricted use, distribution, and reproduction in any medium, provided the original work is properly cited.

\begin{abstract}
Background. The prevalence of gestational diabetes mellitus (GDM) has been dramatically increasing worldwide. The aims of this study were to examine associations of GDM with pregnancy outcomes in Chinese urban women and to evaluate the interaction between GDM and other major risk factors for the risk of adverse pregnancy outcomes. Methods. A retrospective analysis included 8844 women who delivered live singletons at $\geq 28$ weeks of gestation between June 2012 and March 2013 among Chinese urban women. Structured questionnaires were used to collect information on demographic characteristics, lifestyle behavior, medical history, and pregnancy outcomes. The diagnosis of GDM was made between 24 and 28 gestational weeks according to the International Association of Diabetes and Pregnancy Study Groups criteria. Logistic regression models were used to assess the association of GDM with pregnancy outcomes and to examine the interaction between GDM and other major risk factors including maternal age, prepregnancy body mass index, and gestational weight gain for the risk of pregnancy outcomes. Results. 13.9\% of women were diagnosed with GDM. We found that GDM was associated with higher risk of cesarean delivery (odds ratio $(\mathrm{OR})=1.69,95 \%$ CI (confidence interval): 1.48-1.92), preterm birth (OR=1.32, 95\% CI: 1.07-1.64), macrosomia $(\mathrm{OR}=1.69,95 \% \mathrm{CI}: 1.34-2.13)$, and large for gestational age (LGA, OR $=1.43,95 \% \mathrm{CI}$ : 1.18-1.73) after adjustment for potential confounders. We also observed the interaction between GDM and maternal age for the risk of cesarean delivery ( $P$ for interaction $=0.025)$, and the OR of GDM for cesarean delivery was 1.71 (95\% CI: 1.49-1.97) among women aged less than 35 years. Conclusions. GDM was associated with an increased risk of cesarean delivery, preterm birth, macrosomia, and LGA in Chinese urban women, and there was an interaction between GDM and maternal age for the risk of cesarean delivery.
\end{abstract}

\section{Introduction}

GDM is a medical condition of hyperglycemia or glucose intolerance with the first diagnosis in the second or third trimester of pregnancy that is not clearly overt disease prior to gestation [1]. GDM has become a major health concern in pregnant women, and its prevalence has been dramatically increasing worldwide. According to the data from the International Diabetes Federation, GDM was estimated to affect $13.2 \%$ of live births in 2019 [2], while the prevalence of GDM ranges from $9.3 \%$ to $19.7 \%$ in different areas of China and the societal economic burden of GDM was $\$ 5.59$ billion in 2015 at the Chinese national level [3-6]. GDM has been associated with a number of short- and long-term perinatal outcomes not only for the mothers but also for their offspring. For the mothers, GDM is associated with adverse pregnancy complications and outcomes including pregnancy-induced hypertension (PIH), preeclampsia, and cesarean delivery, as well as the lifetime risk of type 2 diabetes and metabolic syndrome [7-9]. For their offspring, it may increase the risk of macrosomia, large for gestational age (LGA), birth injury, neonatal hypoglycemia, and diabetes and subsequently diabetes and obesity later in life $[8,9]$.

As major risk factors for GDM, maternal age, prepregnancy body mass index (BMI), and weight gain during pregnancy were also found to be associated with the risk of adverse maternal and infant outcomes [10]. The average age at childbearing has been rising steadily with the fertility 
rate nearly doubling among women aged above 35 years in recent decades, and women giving birth at advanced maternal age have higher risks of a range of pregnancy complications such as gestational hypertensive disorders, GDM, postpartum haemorrhage, cesarean delivery, and preterm birth $[11,12]$. Apart from maternal age, prepregnancy overweight and obesity, affecting $6 \sim 24 \%$ of Chinese women of childbearing age, have an important influence on both GDM and other maternal and neonatal outcomes such as cesarean delivery, GDM, PIH, neonatal adiposity, and low Apgar scores $[10,13,14]$. Our previous research also reported the associations between prepregnancy overweight and obesity and higher risk of macrosomia and LGA [10]. Furthermore, excessive gestational weight gain (GWG) may contribute to increased risk of cesarean delivery, macrosomia, LGA, hypertensive diseases of pregnancy, and childhood obesity for the offspring, whereas low GWG has been associated with elevated rates of SGA, low birth weight, and preterm birth $[15,16]$. Although several studies examined the association of the forementioned risk factors with adverse perinatal outcomes, little is known about the interactive effect between GDM and other risk factors on adverse pregnancy outcomes in urban Chinese women.

Therefore, the objective of the present study was to evaluate associations of GDM with adverse pregnancy outcomes in Chinese urban women and further assess the associations stratified by maternal age, prepregnancy BMI, and GWG categories, and we also examined the interaction between GDM and maternal age and prepregnancy BMI and GWG for the risk of adverse pregnancy outcomes, respectively.

\section{Materials and Methods}

2.1. Study Design and Participants. This multicenter, retrospective cohort study of postpartum women was conducted in 14 clinical centers located in urban areas of China from June 2012 to March 2013. Details of this study have been described elsewhere [10]. Briefly, we recruited postpartum women aged 18 years or above with a gestational age of $28-42$ weeks and who gave live birth during the $10^{\text {th }}-19^{\text {th }}$ of the last month of every quarter in order to control for seasonal variations. Among 9152 participants with full medical records, 308 women with pregestational diabetes $(n=37)$, multiple gestation $(n=222)$, pregestational diabetes and multiple gestation $(n=1)$, and preconception history of severe heart disease or chronic renal disease $(n=48)$ were excluded. Overall, the present analysis was restricted to 8844 deliveries. This study was approved by the institutional review board of Peking University First Hospital, and all participants provided written informed consent.

2.2. Data Collection. Information on demographic characteristics, lifestyle behavior, medical history of pregnancy, and pregnancy outcomes was collected by using a structured questionnaire after delivery. In-person interviews were conducted to collect information on demographic characteristics such as age, education, employment, and annual household income, as well as lifestyle behavior including drinking and passive smoking during pregnancy, and clinical data such as medical history and pregnancy outcomes was extracted from the medical records.

Gestational age at delivery was determined from the date of the last menstrual period to the date of delivery and expressed in the week after the last menstrual period. If the date was uncertain, ultrasonography was used to determine gestational age. Weight and height at the first antenatal visit prior to the $13^{\text {th }}$ gestational week and weight at the last antenatal visit within 2 weeks before delivery or the time of delivery were extracted from the medical records. Prepregnancy BMI was calculated as the weight in kilograms divided by the square of height measured in meters and classified into three groups according to the Chinese standard [17]: underweight $\left(\mathrm{BMI}<18.5 \mathrm{~kg} / \mathrm{m}^{2}\right)$, normal weight $\left(18.5 \mathrm{~kg} / \mathrm{m}^{2} \leq \mathrm{BMI}<24 \mathrm{~kg} / \mathrm{m}^{2}\right)$, and overweight and obese $\left(B M I \geq 24 \mathrm{~kg} / \mathrm{m}^{2}\right)$. The GWG was calculated by subtracting the weight measured at the first antenatal visit from the final weight measured at the last antenatal visit or the time of delivery. All participants were divided into three groups according to GWG, defined by the $25^{\text {th }}$ and $75^{\text {th }}$ percentile of GWG (12.0 and $19.0 \mathrm{~kg})$ : lower $(\mathrm{GWG}<12 \mathrm{~kg})$, middle $(12 \mathrm{~kg} \leq \mathrm{GWG}<19 \mathrm{~kg})$, and higher $(\geq 19 \mathrm{~kg})$.

2.3. Diagnosis of GDM. According to the International Association of Diabetes and Pregnancy Study Groups (IADPSG) criteria, the diagnosis of GDM should be made when any one of the $75 \mathrm{~g}$ oral glucose tolerance test value met or exceeded $5.1 \mathrm{mmol} / \mathrm{L}$ at $0 \mathrm{~h}, 10.0 \mathrm{mmol} / \mathrm{L}$ at $1 \mathrm{~h}$, and $8.5 \mathrm{mmol} / \mathrm{L}$ at $2 \mathrm{~h}$ when performed between 24 and 28 gestational weeks [18].

2.4. Definition of Adverse Pregnancy Outcomes. The main outcomes of the study were cesarean delivery, preterm birth, low birth weight, small for gestational age (SGA), macrosomia, and LGA. Preterm birth was defined as all birth before 37 completed weeks or before 259 completed days since the first day of a woman's last menstrual period [19]. Low birth weight was defined as neonatal birth weight $<2500 \mathrm{~g}$ and macrosomia as birth weight $\geq 4000 \mathrm{~g}$, respectively. LGA and SGA were indicated by birth weight less than and greater than the $10^{\text {th }}$ and $90^{\text {th }}$ percentile, respectively, for the same gestational age by sex, according to the Chinese neonatal birth weight curve [20].

2.5. Statistical Analysis. Demographic characteristics and pregnancy outcomes were expressed as numbers and frequency distributions for categorical variables or median and interquartile range for continuous variables. To compare between groups, the chi-squared test and Mann-Whitney $U$ test were performed for categorical variables and skewed distributed continuous variables, respectively. Logistic regression models were conducted to estimate odds ratios (ORs) and their 95\% confidence intervals (CIs) of pregnancy outcomes and GDM and interaction between GDM and maternal age/prepregnancy BMI/GWG groups. Models were adjusted for maternal age (continuous), education (high school and below, college or graduate school), employment (unemployed, employed), annual household income (<10000 RMB, 10000$20000 \mathrm{RMB}$, and <20000 RMB), drinking during pregnancy (yes, no), passive smoking during pregnancy (yes, no), 
TABLE 1: Characteristics of participants by gestational diabetes mellitus.

\begin{tabular}{|c|c|c|c|c|}
\hline & All & GDM & Non-GDM & $P$ value \\
\hline Participants, $n(\%)$ & 8844 & $1229(13.9)$ & $7615(86.1)$ & - \\
\hline Maternal age (year), median (IQR) & $28(26,31)$ & $29(26,32)$ & $28(26,30)$ & $<0.001$ \\
\hline Maternal age, $n(\%)$ & & & & $<0.001$ \\
\hline$<35$ years & $8095(91.5)$ & $1074(87.4)$ & $7021(92.2)$ & \\
\hline$\geq 35$ years & $749(8.5)$ & $155(12.6)$ & $594(7.8)$ & \\
\hline Education, $n(\%)$ & & & & 0.801 \\
\hline High school and below & $3346(37.8)$ & $461(37.5)$ & $2885(37.9)$ & \\
\hline College or graduate school & $5498(62.2)$ & $768(62.5)$ & $4730(62.1)$ & \\
\hline Employment, $n(\%)$ & & & & 0.304 \\
\hline Unemployed & $6456(73.0)$ & $912(74.2)$ & $5544(72.8)$ & \\
\hline Employed & $2388(27.0)$ & $317(25.8)$ & $2071(27.2)$ & \\
\hline Annual household income (RMB), $n(\%)$ & & & & 0.874 \\
\hline$<10000$ & $7071(80.0)$ & $980(79.7)$ & $6091(80.0)$ & \\
\hline $10001-20000$ & $1288(14.6)$ & $184(15.0)$ & $1104(14.5)$ & \\
\hline$>20000$ & $485(5.4)$ & $65(5.3)$ & $420(5.5)$ & \\
\hline Drinking during pregnancy, $n(\%)$ & & & & 0.014 \\
\hline No & $8607(97.3)$ & $1209(98.4)$ & $7398(97.2)$ & \\
\hline Yes & $237(2.7)$ & $20(1.6)$ & $217(2.8)$ & \\
\hline Passive smoking during pregnancy, $n(\%)$ & & & & 0.014 \\
\hline No & $7059(79.8)$ & $949(77.2)$ & $6110(80.2)$ & \\
\hline Yes & $1785(20.2)$ & $280(22.8)$ & $1505(19.8)$ & \\
\hline Prepregnancy BMI categories, $n(\%)$ & & & & $<0.001$ \\
\hline Underweight & $7732(87.4)$ & $963(78.4)$ & $6769(88.9)$ & \\
\hline Normal weight & $946(10.7)$ & $204(16.6)$ & $742(9.7)$ & \\
\hline Overweight/obesity & $166(1.9)$ & $62(5.0)$ & $104(1.4)$ & \\
\hline Parity, $n(\%)$ & & & & 0.136 \\
\hline Primiparous & $7237(81.8)$ & $987(80.3)$ & $6250(82.1)$ & \\
\hline Multiparous & $1607(18.2)$ & $242(19.7)$ & $1365(17.9)$ & \\
\hline Use of ART, $n(\%)$ & & & & 0.020 \\
\hline No & $8680(98.2)$ & $1196(97.3)$ & $7484(98.3)$ & \\
\hline Yes & $164(1.8)$ & $33(2.7)$ & $131(1.7)$ & \\
\hline Folic acid supplementation, $n(\%)$ & & & & 0.237 \\
\hline No & $4067(46.0)$ & $546(44.4)$ & $3521(46.2)$ & \\
\hline Yes & $4777(54.0)$ & $683(44.6)$ & $4094(53.8)$ & \\
\hline Gestational age at delivery (week), median (IQR) & $39(38,40)$ & $39(38,39)$ & $39(38,40)$ & $<0.001$ \\
\hline Gestational weight gain $(\mathrm{kg})$ & & & & $<0.001$ \\
\hline Lower & $1282(14.5)$ & $245(19.9)$ & $1037(13.6)$ & \\
\hline Middle & $6367(72.0)$ & $833(67.8)$ & $5534(72.7)$ & \\
\hline Higher & $1195(13.5)$ & $151(12.3)$ & $1044(13.7)$ & \\
\hline
\end{tabular}

Abbreviations: ART: assisted reproductive technology; BMI: body mass index; GDM: gestational diabetes mellitus. Values are the median (interquartile range) or $n(\%)$.

prepregnancy BMI categories (underweight, normal weight, and overweight/obese), parity (primiparous, multiparous), use of assisted reproductive technology (ART; yes, no), folic acid supplementation (yes, no), gestational age at delivery (continuous, except for the outcome of preterm birth), and GWG categories (lower, middle, and higher). The non-GDM group was used as the reference group. Stratified analyses were performed according to maternal age groups ( $<35$ years, $\geq 35$ years), prepregnancy BMI categories (underweight, normal weight, and overweight/obese), and GWG categories (lower, middle, and higher). Analyses were carried out using SAS software version 9.2 (SAS Institute, Cary, NC). All $P$ values are two-sided, and statistical significance was defined as $P<0.05$.

\section{Results}

3.1. Participant Characteristics. Among 8844 postpartum women, 1229 (13.9\%) were diagnosed with GDM. The 
average age of study participants was 28.7 years. Distribution of GDM status by study centers is shown in Table S1. Table 1 shows demographic characteristics of participants according to GDM status. Women with GDM had a higher likelihood of advanced maternal age ( $\geq 35$ years), prepregnancy overweight and obesity, and use of ART than non-GDM counterparts (all $P<0.05$ ). Compared to those unaffected by GDM, women with GDM were more likely to be exposed to passive smoking during pregnancy and less likely to drink during pregnancy and to be in the middle group of GWG (all $P<0.05$ ).

3.2. Prevalence of Pregnancy Outcomes by GDM. Figure 1 and Table S2 illustrate the prevalence of adverse pregnancy outcomes by GDM status. Women with GDM had a higher likelihood of cesarean delivery ( $60.0 \%$ vs. $45.8 \%)$, macrosomia (9.4\% vs. $6.1 \%)$, and LGA (14.0\% vs. 9.7\%) than those who did not have GDM (all $P<0.001$ ). However, the prevalence of preterm birth, low birth weight, and SGA was comparable between the two groups (all $P>0.05$ ).

3.3. Associations of GDM with Pregnancy Outcomes. Table 2 shows associations of adverse pregnancy outcomes with GDM status. After adjustment for potential confounders, GDM was associated with a higher risk for cesarean delivery $(\mathrm{OR}=1.69,95 \% \mathrm{CI}: 1.48-1.92)$, preterm birth $(\mathrm{OR}=1.32$, 95\% CI: $1.07-1.64)$, macrosomia (OR $=1.69,95 \% \mathrm{CI}: 1.34-$ 2.13), and LGA (OR = 1.43, 95\% CI: 1.18-1.73).

3.4. The Effect of the Interaction between GDM and Maternal Age on Pregnancy Outcomes. There was a significantly statistical interaction between GDM and maternal age ( $<35$ years, $\geq 35$ years) for the risk of cesarean delivery after adjustment for potential confounders ( $P$ for interaction $=0.025$, Table 3 ). The prevalence of cesarean delivery was higher in women with GDM than those who did not have GDM regardless of maternal age (age < 35 years: $58.5 \%$ vs. $44.3 \%$; age $\geq 35$ years: $67.7 \%$ vs. $64.3 \%)$. Among women of age $<35$ years, the OR of GDM for cesarean delivery was in 1.71 (95\% CI: 1.49-1.97) after adjustment for potential confounders, while GDM was not significantly associated with the risk of cesarean delivery among those aged 35 years and above. In addition, we further performed the combined analysis to evaluate the effect of GDM and maternal age on the risk of cesarean delivery (Figure 2). Compared to those aged $<35$ years and having no GDM, women with GDM and aged $\geq 35$ years had a 2.10 -fold odds of cesarean delivery ( $\mathrm{OR}=2.10,95 \% \mathrm{CI}$ : 1.48-2.93).

We also examined the relationships between GDM and pregnancy outcomes by prepregnancy BMI and GWG categories, respectively. Results were generally consistent pertaining to associations of GDM with the risk of pregnancy outcomes across different prepregnancy BMI (Table S3) and GWG groups (Table S4).

\section{Discussion}

In this retrospective cohort study of Chinese urban women, $13.9 \%$ of women were diagnosed with GDM. We found that GDM was associated with a higher risk of cesarean delivery, preterm birth, macrosomia, and LGA after adjustment for

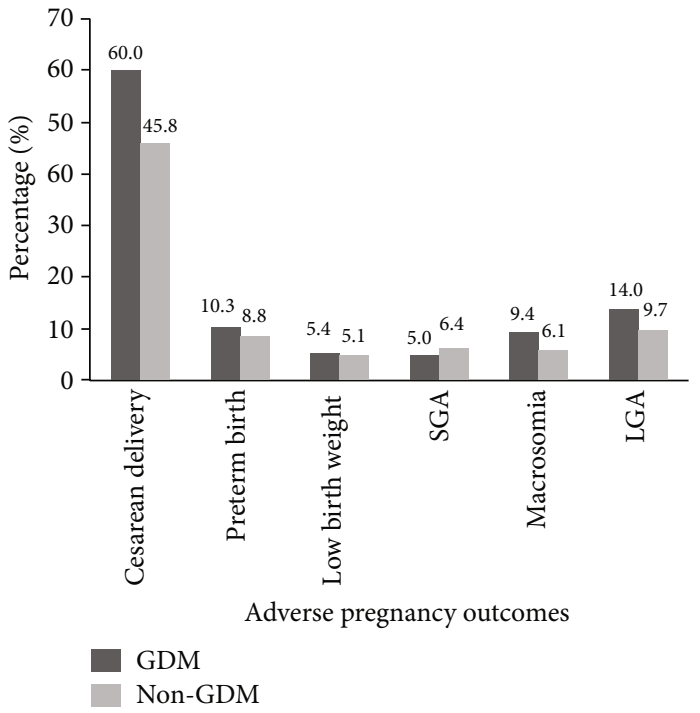

FIgURe 1: Percentage of adverse pregnancy outcomes by gestational diabetes mellitus. Abbreviations: GDM: gestational diabetes mellitus; SGA: small for gestational age; LGA: large for gestational age.

potential confounders. We also observed the interaction between GDM and maternal age for the risk of cesarean delivery.

The positive associations of GDM with increased risk of macrosomia and LGA have been reported in different populations, which is in accordance with our study [21-23]. A retrospective cohort study enrolling more than 21000 Thai women indicated that the rate of macrosomia was significantly higher in the GDM than in the control, and GDM was associated with a $48 \%$ increased risk of macrosomia [21]. In addition, the Hyperglycemia and Adverse Pregnancy Outcome (HAPO) study conducted in 15 centers in nine countries demonstrated the continuous positive associations between maternal glucose concentrations and birth weight even among those who had plasma glucose previously considered normal for pregnancy [24]. Previous randomized controlled trials also implied that glycemic control through dietary intervention, physical activity, and/or insulin therapy was able to reduce the risk of macrosomia and LGA $[25,26]$. Recently, a prospective cohort study based on a US population suggested that GDM was associated with a larger fetal size that started at gestational week 20 and became significant at gestational week 28 , indicating that interventions to mitigate fetal overgrowth related to GDM should be initiated before the diagnosis of GDM [27]. The mechanisms and pathways for the relationship between maternal hyperglycemia and neonatal birth weight are not well described. It might be explained that maternal hyperglycemia and insulin resistance might cause fetal hyperinsulinemia and thus enhanced the utilization of nutrients and consequently leading to fetal overgrowth and adiposity $[23,28]$.

Another finding of our study is that GDM was associated with an increased risk of preterm birth. Prior research on the relationship between GDM and risk of preterm birth has been still controversial. Similar to our findings, a population study focusing on more than 327,000 singleton pregnancies 
TABLE 2: ORs (95\% CIs) for pregnancy outcomes by gestational diabetes mellitus.

\begin{tabular}{|c|c|c|c|c|c|}
\hline & \multirow{2}{*}{ Case/noncase } & \multicolumn{2}{|c|}{ Model 1} & \multicolumn{2}{|c|}{ Model 2} \\
\hline & & OR (95\% CI) & $P$ value & OR (95\% CI) & $P$ value \\
\hline Cesarean delivery & $4227 / 4617$ & $1.75(1.54,1.99)$ & $<0.001$ & $1.69(1.48,1.92)$ & $<0.001$ \\
\hline Preterm birth* & $800 / 8044$ & $1.37(1.11,1.70)$ & 0.004 & $1.32(1.07,1.64)$ & 0.011 \\
\hline Low birth weight & $454 / 8390$ & $1.17(0.88,1.56)$ & 0.276 & $0.86(0.59,1.25)$ & 0.422 \\
\hline SGA & $548 / 8296$ & $0.76(0.57,1.01)$ & 0.062 & $0.75(0.56,1.01)$ & 0.051 \\
\hline Macrosomia & $579 / 8265$ & $1.58(1.26,1.97)$ & $<0.001$ & $1.69(1.34,2.13)$ & $<0.001$ \\
\hline LGA & $908 / 7936$ & $1.50(1.24,1.81)$ & $<0.001$ & $1.43(1.18,1.73)$ & $<0.001$ \\
\hline
\end{tabular}

Abbreviations: CI: confidence interval; OR: odds ratio; SGA: small for gestational age; LGA: large for gestational age. Model 1 was adjusted for demographic characteristics including maternal age, education, employment, annual household income, and study centers. Model 2 was further adjusted for drinking during pregnancy, passive smoking during pregnancy, prepregnancy body mass index categories, parity, use of assisted reproductive technology, folic acid supplementation, gestational age at delivery, and gestational weight gain categories. Preterm birth was not adjusted for gestational age at delivery*.

TABLE 3: ORs (95\% CIs) for pregnancy outcomes by gestational diabetes mellitus, stratified by maternal age.

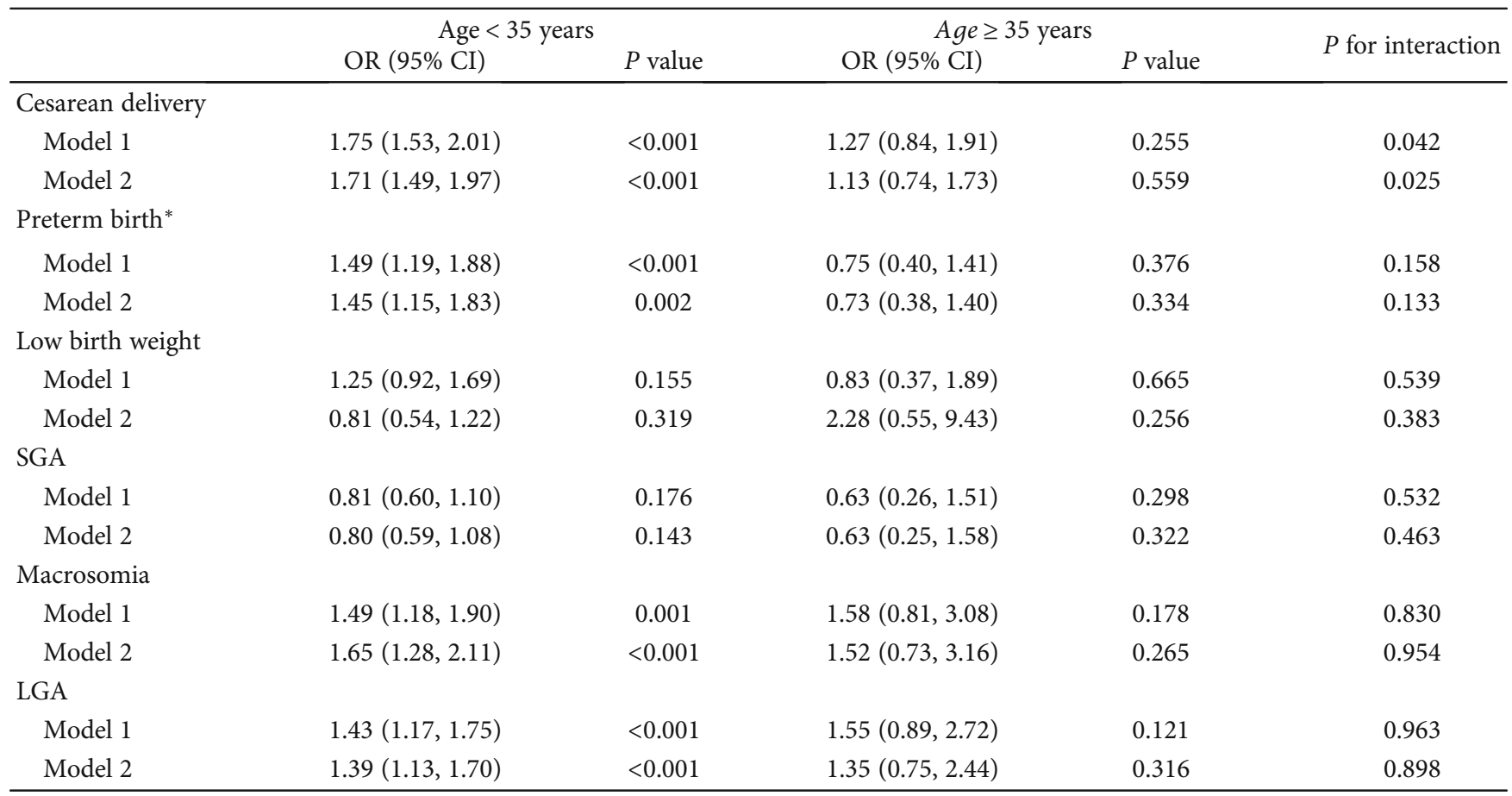

Abbreviations: CI: confidence interval; OR: odds ratio; SGA: small for gestational age; LGA: large for gestational age. Model 1 was adjusted for demographic characteristics including maternal age, education, employment, annual household income, and study centers. Model 2 was further adjusted for drinking during pregnancy, passive smoking during pregnancy, prepregnancy body mass index categories, parity, use of assisted reproductive technology, folic acid supplementation, gestational age at delivery, and gestational weight gain categories. Preterm birth was not adjusted for gestational age at delivery*.

in Canada indicated that GDM was associated with an increased risk of spontaneous preterm and labor-induced preterm in singleton pregnancies [29]. Moreover, a population-based study conducted in northeastern Germany reported that GDM resulted in a higher percentage of late preterm infants with a gestational age of 32-36 weeks (11.1\% vs. $7.0 \%)$ [30]. However, several retrospective studies found no increased risk for preterm delivery in women having GDM in comparison to non-GDM counterparts [31, 32]. Therefore, further research is needed to explore the relationship and underlying mechanisms between GDM and the risk of preterm birth.
In addition, we also observed that GDM was associated with a $62 \%$ increased risk of cesarean delivery. Consistent with our findings, the data from the Massachusetts Pregnancy to Early Life Longitudinal Data System has shown that women with GDM during one of two sequential pregnancies had an increased risk of cesarean delivery [33]. A large-scale observational national study in France found that the risk of cesarean delivery was increased in women with GDM compared with the nondiabetic population in deliveries after 28 weeks [34]. Another observational study conducted at 15 medical centers in Beijing demonstrated that GDM women had an elevated risk of cesarean delivery compared with 


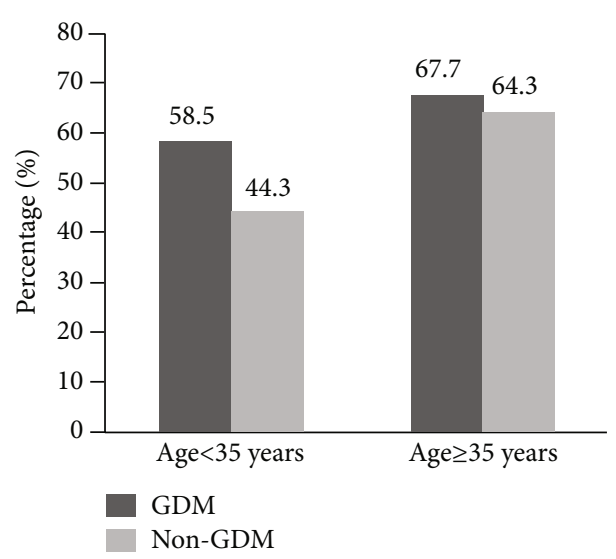

(a)

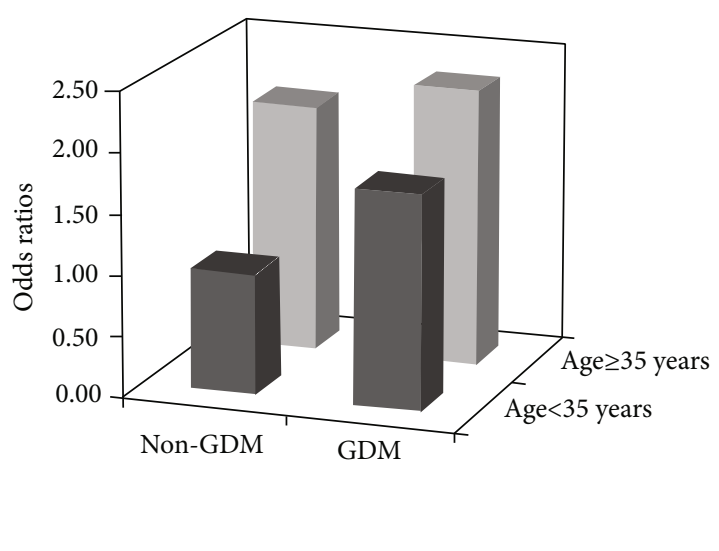

(b)

FIgUre 2: Associations of gestational diabetes mellitus and maternal age with the risk of cesarean delivery. (a) Percentage of gestational diabetes mellitus by maternal age groups. Values are $n$ (\%). (b) Adjusted odds ratios for cesarean delivery according to different gestational diabetes mellitus and maternal age groups. Values are odds ratios. Adjusted for maternal age, education, employment, annual household income, study centers, drinking during pregnancy, passive smoking during pregnancy, prepregnancy body mass index categories, parity, use of assisted reproductive technology, folic acid supplementation, gestational age at delivery, and gestational weight gain categories. Abbreviations: GDM: gestational diabetes mellitus.

normal blood glucose women [35]. The HAPO study revealed that the odds ratio for primary cesarean section increased across categories of maternal glycemia with an OR of 1.86 in the highest category of 1-hour plasma glucose [24]. The association between GDM and the increased risk of cesarean delivery may be influenced by two aspects: on the one hand, maternal hyperglycemia might contribute to fetal overgrowth and large neonatal birth weight, which could lead to the higher risk of cesarean delivery; on the other hand, women developing GDM may affect clinical decisionmaking due to the higher rates of abnormalities in labor, birth trauma, and fetal distress, which might increase the risk of cesarean delivery [36]. However, potential mechanisms need further exploration in future research.

One interesting finding of our study is that we found a significant interaction between GDM and maternal age in relation to the risk of cesarean delivery. Specifically, we observed that women aged 35 years and above and having GDM had a 2.1-fold increased risk of cesarean delivery compared to those who were under 35 years and did not have GDM. Moreover, the stratified analysis of our study indicated that GDM was much more important in women aged less than 35 years than in those 35 years and above among Chinese urban women. In contrast to our finding, a previous retrospective study focusing on 880 Portuguese women also found the interaction between GDM and maternal age for cesarean delivery by reporting that women older than 35 years presented a higher association between GDM and nonelective cesarean delivery than women younger than 35 years [36]. Differences in findings may be partly due to ethnicity disparities and focus on different types of cesarean delivery. Overall, our finding emphasizes the important public health implications of glycemic control by lifestyle intervention among pregnant women developing GDM, which is particularly crucial in Chinese urban women under 35 years.

The main strengths of our study include a relatively large sample size, using the IADPSG criteria for GDM diagnosis that is reported to be more robust for diagnosis of GDM, and its influence on adverse maternal and perinatal outcomes [37], further assessing the interaction of GDM and other major factors for adverse pregnancy outcomes and adjusting for potential confounding factors comprehensively. However, our study has a few limitations. Firstly, we did not distinguish between emergency and elective cesarean deliveries and did not differentiate spontaneous and indicated preterm birth. Secondly, our data included only the Chinese Han population, and it is unclear whether the results can be extrapolated to women of other ethnic groups. Lastly, we did not collect blood specimens and specific plasma glucose levels such as fasting and 1-hour and 2-hour plasma glucose levels, so we were unable to examine associations of pregnancy outcomes with certain glycemic parameters.

\section{Conclusions}

In summary, our study indicated that GDM was associated with a higher risk of cesarean delivery, preterm birth, macrosomia, and LGA. We also observed the interaction between GDM and maternal age for the risk of cesarean delivery. Our findings emphasize the negative impact of GDM on pregnancy outcomes among Chinese urban women, particularly in those below 35 years old.

\section{Data Availability}

The data used to support the findings of this study are available from the corresponding author upon request.

\section{Disclosure}

This manuscript has been posted as a preprint on Research Square. 


\section{Conflicts of Interest}

The authors declared no conflict of interest.

\section{Acknowledgments}

This study was funded by the Scientific Research Seed Fund of Peking University First Hospital Project (2019SF37). We thank all participants and investigators and all staff of the medical record section in 14 hospitals.

\section{Supplementary Materials}

Table S1: distribution of gestational diabetes mellitus by study centers. Table S2: pregnancy outcomes by gestational diabetes mellitus. Table S3: adjusted ORs (95\% CIs) for pregnancy outcomes by gestational diabetes mellitus, stratified by prepregnancy body mass index. Table S4: adjusted ORs (95\% CIs) for pregnancy outcomes by gestational diabetes mellitus, stratified by gestational weight gain. (Supplementary Materials)

\section{References}

[1] American Diabetes Association, "2. Classification and diagnosis of diabetes: standards of medical care in diabetes-2019," Diabetes Care, vol. 42, no. 1, pp. S13-S28, 2019.

[2] C. Jin, L. Lin, N. Han et al., "Effects of dynamic change in fetuin-A levels from the first to the second trimester on insulin resistance and gestational diabetes mellitus: a nested casecontrol study," BMJ Open Diabetes Research \& Care, vol. 8, no. 1, p. e000802, 2020.

[3] T. Xu, L. Dainelli, K. Yu et al., "The short-term health and economic burden of gestational diabetes mellitus in China: a modelling study," BMJ Open, vol. 7, no. 12, article e018893, 2017.

[4] J. Leng, P. Shao, C. Zhang et al., "Prevalence of gestational diabetes mellitus and its risk factors in Chinese pregnant women: a prospective population-based study in Tianjin, China," PLoS One, vol. 10, no. 3, article e0121029, 2015.

[5] G. Li, T. Wei, W. Ni et al., "Incidence and risk factors of gestational diabetes mellitus: a prospective cohort study in Qingdao, China," Front Endocrinol (Lausanne), vol. 11, p. 636, 2020.

[6] W. W. Zhu, H. X. Yang, C. Wang, R. N. Su, H. Feng, and A. Kapur, "High prevalence of gestational diabetes mellitus in Beijing: effect of maternal birth weight and other risk factors," Chinese Medical Journal, vol. 130, no. 9, pp. 10191025, 2017.

[7] K. W. Lee, S. M. Ching, V. Ramachandran et al., "Prevalence and risk factors of gestational diabetes mellitus in Asia: a systematic review and meta-analysis," BMC Pregnancy and Childbirth, vol. 18, no. 1, p. 494, 2018.

[8] P. Saravanan, "Gestational diabetes: opportunities for improving maternal and child health," The Lancet Diabetes and Endocrinology, vol. 8, no. 9, pp. 793-800, 2020.

[9] P. Damm, A. Houshmand-Oeregaard, L. Kelstrup, J. Lauenborg, E. R. Mathiesen, and T. D. Clausen, "Gestational diabetes mellitus and long-term consequences for mother and offspring: a view from Denmark," Diabetologia, vol. 59, no. 7, pp. 1396-1399, 2016.
[10] X. Wang, X. Zhang, M. Zhou, and J. Juan, "Association of prepregnancy body mass index, rate of gestational weight gain with pregnancy outcomes in Chinese urban women," Nutrition \& Metabolism (London), vol. 16, no. 1, p. 54, 2019.

[11] K. E. Fitzpatrick, D. Tuffnell, J. J. Kurinczuk, and M. Knight, "Pregnancy at very advanced maternal age: a UK populationbased cohort study," BJOG, vol. 124, no. 7, pp. 1097-1106, 2017.

[12] T. Kato, T. Yorifuji, M. Yamakawa et al., "Association of maternal age with child health: a Japanese longitudinal study," PLoS One, vol. 12, no. 2, article e0172544, 2017.

[13] J. Stang and L. G. Huffman, "Position of the academy of nutrition and dietetics: obesity, reproduction, and pregnancy outcomes," Journal of the Academy of Nutrition and Dietetics, vol. 116, no. 4, pp. 677-691, 2016.

[14] T. H. Hung and T. T. Hsieh, "Pregestational body mass index, gestational weight gain, and risks for adverse pregnancy outcomes among Taiwanese women: a retrospective cohort study," Taiwanese Journal of Obstetrics \& Gynecology, vol. 55, no. 4, pp. 575-581, 2016.

[15] Life Cycle Project-Maternal Obesity and Childhood Outcomes Study Group, E. Voerman, S. Santos et al., "Association of gestational weight gain with adverse maternal and infant outcomes," JAMA, vol. 321, no. 17, pp. 1702-1715, 2019.

[16] R. F. Goldstein, S. K. Abell, S. Ranasinha et al., "Association of gestational weight gain with maternal and infant outcomes: a systematic review and meta-analysis," JAMA, vol. 317, no. 21, pp. 2207-2225, 2017.

[17] Y. Wang, J. Mi, X. Y. Shan, Q. J. Wang, and K. Y. Ge, "Is China facing an obesity epidemic and the consequences? The trends in obesity and chronic disease in China," International Journal of Obesity, vol. 31, no. 1, pp. 177-188, 2007.

[18] International Association of Diabetes and Pregnancy Study Groups Consensus Panel, B. E. Metzger, S. G. Gabbe et al., "International association of diabetes and pregnancy study groups recommendations on the diagnosis and classification of hyperglycemia in pregnancy," Diabetes Care, vol. 33, no. 3, pp. 676-682, 2010.

[19] C. M. Chen, "Overview of obesity in Mainland China," Obesity Reviews, vol. 9, Suppl 1, pp. 14-21, 2008.

[20] H. Li, J. Zheng, H. Wang et al., "Maternal cosmetics use during pregnancy and risks of adverse outcomes: a prospective cohort study," Scientific Reports, vol. 9, no. 1, p. 8030, 2019.

[21] S. Srichumchit, S. Luewan, and T. Tongsong, "Outcomes of pregnancy with gestational diabetes mellitus," International Journal of Gynaecology and Obstetrics, vol. 131, no. 3, pp. 251-254, 2015.

[22] G. Seghieri, G. Di Cianni, M. Seghieri et al., "Risk and adverse outcomes of gestational diabetes in migrants: a population cohort study," Diabetes Research and Clinical Practice, vol. 163, p. 108128, 2020.

[23] P. M. Catalano, H. D. McIntyre, J. K. Cruickshank et al., "The hyperglycemia and adverse pregnancy outcome study: associations of GDM and obesity with pregnancy outcomes," Diabetes Care, vol. 35, no. 4, pp. 780-786, 2012.

[24] HAPO Study Cooperative Research Group, B. E. Metzger, L. P. Lowe et al., "Hyperglycemia and adverse pregnancy outcomes," The New England Journal of Medicine, vol. 358, no. 19, pp. 1991-2002, 2008.

[25] M. B. Landon, C. Y. Spong, E. Thom et al., "A multicenter, randomized trial of treatment for mild gestational diabetes," The 
New England Journal of Medicine, vol. 361, no. 14, pp. 13391348, 2009.

[26] X. Yang, H. Tian, F. Zhang et al., “A randomised translational trial of lifestyle intervention using a 3-tier shared care approach on pregnancy outcomes in Chinese women with gestational diabetes mellitus but without diabetes," Journal of Translational Medicine, vol. 12, no. 1, p. 290, 2014.

[27] M. Li, S. N. Hinkle, K. L. Grantz et al., "Glycaemic status during pregnancy and longitudinal measures of fetal growth in a multi-racial US population: a prospective cohort study," The Lancet Diabetes and Endocrinology, vol. 8, no. 4, pp. 292300, 2020.

[28] K. Kc, S. Shakya, and H. Zhang, "Gestational diabetes mellitus and macrosomia: a literature review," Annals of Nutrition \& Metabolism, vol. 66, Suppl 2, pp. 14-20, 2015.

[29] F. Y. Lai, J. A. Johnson, D. Dover, and P. Kaul, "Outcomes of singleton and twin pregnancies complicated by pre-existing diabetes and gestational diabetes: a population-based study in Alberta, Canada, 2005-11," Journal of Diabetes, vol. 8, no. 1, pp. 45-55, 2016.

[30] G. Domanski, A. E. Lange, T. Ittermann et al., "Evaluation of neonatal and maternal morbidity in mothers with gestational diabetes: a population-based study," BMC Pregnancy and Childbirth, vol. 18, no. 1, p. 367, 2018.

[31] Y. Yogev and O. Langer, "Spontaneous preterm delivery and gestational diabetes: the impact of glycemic control," Archives of Gynecology and Obstetrics, vol. 276, no. 4, pp. 361-365, 2007.

[32] N. M. Nordin, J. W. Wei, N. N. Naing, and E. M. Symonds, "Comparison of maternal-fetal outcomes in gestational diabetes and lesser degrees of glucose intolerance," The Journal of Obstetrics and Gynaecology Research, vol. 32, no. 1, pp. 107114, 2006.

[33] S. Y. Kim, M. Kotelchuck, H. G. Wilson, H. Diop, C. K. Shapiro-Mendoza, and L. J. England, "Prevalence of adverse pregnancy outcomes, by maternal diabetes status at first and second deliveries, Massachusetts, 1998-2007," Preventing Chronic Disease, vol. 12, article E218, 2015.

[34] C. Billionnet, D. Mitanchez, A. Weill et al., "Gestational diabetes and adverse perinatal outcomes from 716,152 births in France in 2012," Diabetologia, vol. 60, no. 4, pp. 636-644, 2017.

[35] G. Song, Y. M. Wei, W. W. Zhu, and H. X. Yang, "Cesarean section rate in singleton primiparae and related factors in Beijing, China," Chinese Medical Journal, vol. 130, no. 20, pp. 2395-2401, 2017.

[36] R. Gorgal, E. Gonçalves, M. Barros et al., "Gestational diabetes mellitus: a risk factor for non-elective cesarean section," The Journal of Obstetrics and Gynaecology Research, vol. 38, no. 1, pp. 154-159, 2012.

[37] S. Todi, H. Sagili, and S. K. Kamalanathan, "Comparison of criteria of International Association of Diabetes and Pregnancy Study Groups (IADPSG) with National Institute for Health and Care Excellence (NICE) for diagnosis of gestational diabetes mellitus," Archives of Gynecology and Obstetrics, vol. 302, no. 1, pp. 47-52, 2020. 